\title{
Safety of bronchoscopic guided percutaneous dilatational tracheostomy in ICU patients in Tabriz City
}

\author{
Mohammad Esmaeil Hejazi*, Mohammadamin Rezazadehsaatlou*, Leila Namvar*, \\ Armin Sadeghi*, Veghar Hejazi*, Yasin Hejazi*, and Katrin Buyuk Aghazadeh*
}

\section{ABSTRACT}

\section{BACKGROUND}

Tracheostomy is one of the most frequently performed procedures in intensive care units (ICU). The widespread attraction of percutaneous dilatational tracheostomy (PDT) is increasing in modern ICU. Bronchoscopic guidance seems to secure the safety of the technique. Multiple studies done to explain characterize differences in complications and cost-effectiveness of open and percutaneous tracheotomy. The objective of this study was to evaluate the benefits of PDT using the Ciaglia technique with bronchoscopic guidance.

\section{METHODS}

A total of 100 elective PDT using the Ciaglia technique with a little modification were performed under flexible fiber optic bronchoscopic guide. The demographic variables were recorded, the underlying cause for patient's referred to the center for PDT, and intraoperative as well as early postoperative complications. Patients followed for several days after tracheostomy for early complications.

\section{RESULTS}

No severe complications related to PDT were noticed during and after the procedure. Three patients had bleeding during incision and two led to subcutaneus hematoma. There were no other complications such as infection, emphysema and puncture of posterior wall.

\section{CONCLUSIONS}

We recommended the use of endoscopic guidance bedside percutaneous tracheostomy using the Ciaglia technique with a little modification because it is safe and simple to do without significant complications. PDT with bronchoscopic guidance is a safe and easy procedure that can be done at the bedside setting.

Keywords: Percutaneous dilatational tracheostomy, bronchoscopy, complication
*Tuberculosis and Lung Disease Research Center,

Tabriz University of Medical Sciences, Imam Reza Hospital, Division of Respiratory and Critical Care Medicine,

Department of Internal Medicine, Tabriz, Iran

\section{Correspondence :}

Katrin BuyukAghazadeh

Tuberculosis and Lung Disease

Research Center,

Tabriz University of Medical

Sciences, Imam Reza Hospital,

Division of Respiratory and Critical

Care Medicine,

Department of Internal Medicine,

Tabriz, Iran

Email: katrinaghazadeh@gmail.com

Date of first submission, August 14 2017

Date of final revised submission,

November 2, 2017

Date of acceptance, November 2, 2017

This open access article is distributed under a Creative Commons AttributionNon Commercial-Share Alike 4.0

International License

Cite this article as: Hejazi ME, Rezazadehsaatlou M, Namvar L, et al. Safety of bronchoscopic guided percutaneous dilatational tracheostomy in ICU patients in Tabriz City. Univ Med 2017;36:173-8. doi: 10.18051/ UnivMed.2017.v36.173-178 


\section{INTRODUCTION}

Tracheostomy is one of the oldest surgical procedures, described in Hindu and Egyptian texts dating back to $1000 \mathrm{BC} \cdot{ }^{(1)}$ Tracheostomy usually performed in patients with difficult weaning from mechanical ventilation or somecatastrophic neurologic disorders, also many laryngeal infectious and neoplastic processes may require a surgical airway. ${ }^{(2,3)}$ To avoid the complications of chronic endotracheal intubation, tracheostomy performed in patients requiring long-term intubation to facilitate pulmonary hygiene and weaning from the ventilator. ${ }^{(4)}$ Other indications include management of upper airway obstruction and copious secretionsand to minimize laryngeal injury. ${ }^{(5)}$ In recent studies the benefits of early tracheostomy in patients who require extended period of mechanical ventilation compared with prolonged trans laryngeal intubation. Percutaneous dilatational tracheostomy (PDT) techniques have been described recently, these techniques providing the clinicians a chance to perform rapidly and easily tracheostomy atthebedside, the complications associated with this new procedurearen't definedyet. ${ }^{(4)}$

Percutaneous dilatational tracheostomy is the standard airway access in critically ill patients who require prolonged mechanical ventilation. ${ }^{(6)}$ Percutaneous dilatational tracheostomy is a rapid, simple, easy to learn and cost-effective procedure. It has many advantages over surgical tracheostomy. Over the years, PDT has evolved as a safe bedside procedure that has enabled a temporary tracheostomy to be inserted by the intensive sits in ICU. ${ }^{(7)}$ Ciaglia et al. ${ }^{(8)}$ in 1985 introduced principle technique of contemporary percutaneous tracheostomy and refined in 1999 as Ciaglia Blue Rhino ${ }^{(9)}$ and 2005 as Ciaglia Blue Dolphin. ${ }^{(10)}$ The Ciaglia Blue Dolphin or its modifications have been used increasingly in different patient populations. ${ }^{(11)}$

Both the surgical tracheostomy (ST) and the PDT strategies provide advantages and disadvantages. Surgical tracheostomy immediately secures the newly established access to the airway. In contrast, the PDT is performed via a small skin incision followed by different dilatational mechanisms and insertion of the tracheal cannula. Consequently, there is disagreement regarding which strategy offers the superior benefit/risk ratio for critically ill patients. A systematic review and meta-analysis to compare percutaneous with surgical strategy for tracheostomy showed that the choice of strategy is primarily made by the preference of the surgeon and not by evidence, as evidence for the strategy with the best parameters of safety is poor and conflicting. ${ }^{(12)}$

Over the past decade, the application of various imaging guidance modalities (flexible bronchoscopy, rigid bronchoscopy, and ultrasound) has further enhanced PDT and extended its use in a variety of patient populations. ${ }^{(13)}$ The use of guidance bronchoscopy in PDT has not been adequately described. This study aimed to evaluate PDT with bronchoscopy guidance similar to Cialgia with a little change in method to shorten the time and reduce the complications.

\section{METHODS}

\section{Study design and subjects}

In this clinical trial we prospectively analyzed the data from 100 patients who underwent tracheostomy during about 1.5 years period (from January 2016 to June 2017).

All patients were from pulmonary ICU of Imam Reza hospital and Shahid Madani Hospital of Tabriz University, Iran. All patients need prolonged mechanical ventilation (more than 7 days) due to medical problems and require an artificial airway to prolonged ventilation, meanage was 59 years old, and a total of 100 patient were included.Inclusion criteria were traditional indication to tracheostomy, along with having none of absolute or relative contraindications to PDT (coagulopathy, high positive end-expiratory pressure (PEEP) or inspired oxygen concentration (FiO2) requirement, difficult anatomy, and hemodynamic instability). The exclusion criteria were obesity and patients with short neck or scar 
in neck and distortion of trachea and large thyroid gland.

\section{Performance of PDT}

All patients sedated with Fentanyl (100-200 $\mathrm{mcg} / \mathrm{IV}$ ) and Midazolam ( 3 to $5 \mathrm{mg} / \mathrm{IV}$ ) then neck is hyperextended by using a shoulder pad. Bronchoscope is inserted true Endotracheal Tube (ETT) and adjusted so that the lower edge of ETT was visible, throughout the procedure the bronchoscope is in this position to see the all steps of procedure. Local anesthesia is done by Lidocaine $2 \%$ ( $3-5 \mathrm{cc}$ ). Transverse incision (1.5 $\mathrm{cm}$ ) is made $2 \mathrm{~cm}$ below cricoid cartilage and curved hemostat is used for separation of subcutaneous tissue. Retraction done until fibromuscular tissue over laying the tracheal rings detected. Needle inserted between the second and third tracheal rings, through the needle J shape guide wire directed toward the carina. The needle removed and a short 14-F dilator inserted over the guidewire then the dilator is removed with $\mathrm{J}$ guidwire in place. Ciaglia blue rhino loaded on an
$8 \mathrm{~F}$ guiding catheter are inserted over the guidewire. Dilator removed leaving the guiding catheter in place then the tracheostomy tube loaded over a dilator inserted in to the trachea and then guiding wire and catheter are removed. The bronchoscope inserted through the tracheostomy tube to ensure the placement of tube then fixation done by band (Figure 1; A: Incision; B: Insertion of guidwire; $\mathrm{C}$ : insertion of small dilator; D: preparation of dilator; $\mathrm{E}$ : tracheostomy replacement; F: tracheostomy fixation). Mean time for the procedure from skin incision to tracheostomy tube placement was 3 minutes.

\section{Assessment of complications}

Complications related to PDT, such as serious complication (cardiopulmonary arrest, trachoesophageal fistula, intermediate complications and posterior tracheal wall perforation) and minor complication (bleeding, tube misplacement and emphysema) were documented.

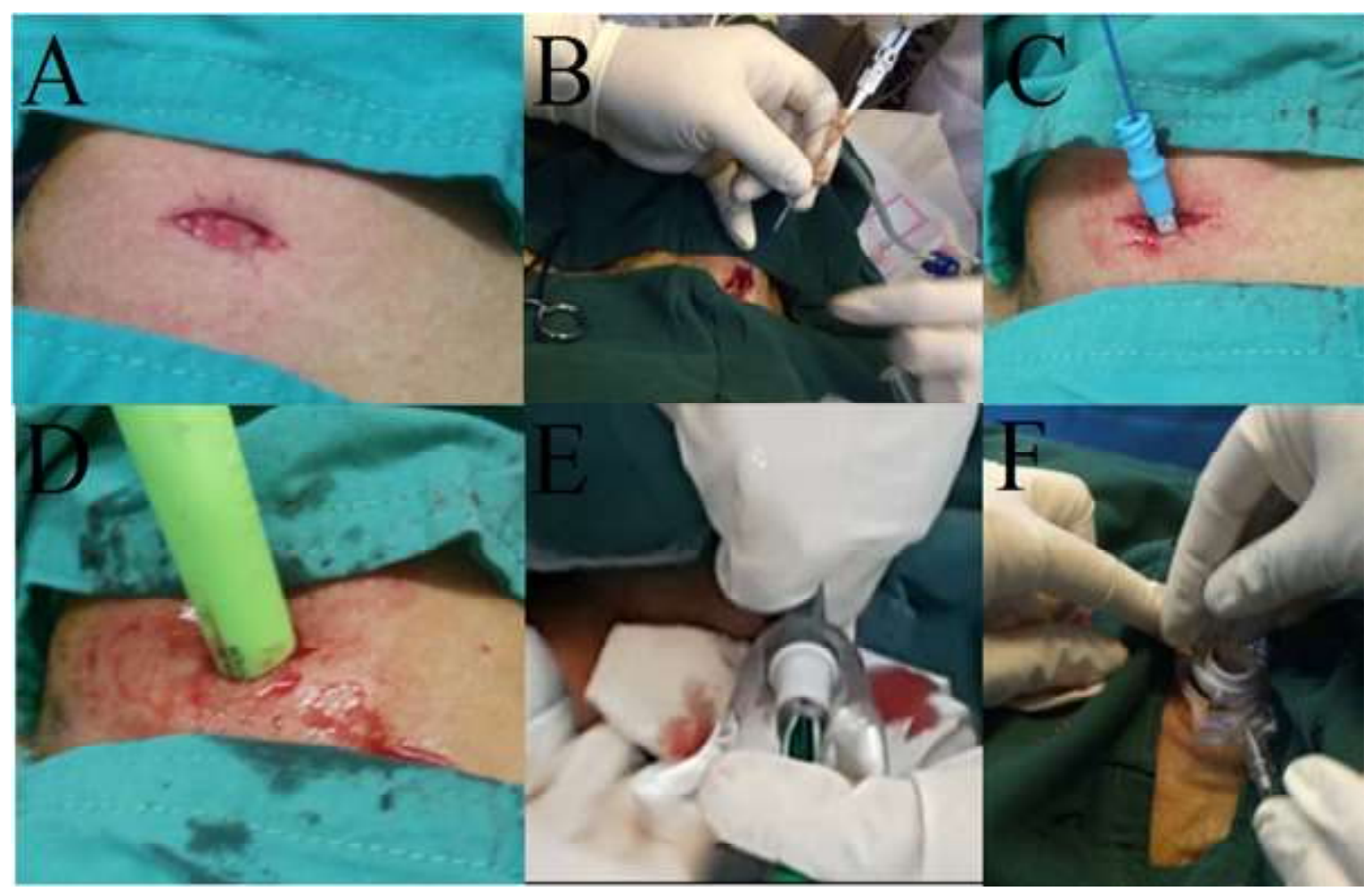

Figure 1. Stages of percutaneous dilatational tracheostomy

A:Incision; B: Insertion of guidwire; C: insertion of small dilator; D: preparation of dilator;

$\mathrm{E}$ : tracheostomy replacement; F: tracheostomy fixation 


\section{Ethical clearance}

This study received ethical clearance from the Health Research Ethics Committee, Faculty of Medicine, Tabriz University of Medical Sciences.All patients had given written consent for surgery (this study is retrospective study).

\section{RESULTS}

From 100 patients (34 female and 66 male with mean age 59.5 years old) who underwent percutaneous tracheostomy, morbidity rate directly attributed to the tracheostomy was $0 \%$. No major early complications were found in all patients. In this technique there was small amount of bleeding (about 3-4 cc) and only one of cases had massive bleeding that caused stop of procedure and patient referred to surgical tracheostomy and three patient had minor bleeding from dilatation site that controlled without any complication; meanwhile of these 3 cases two patients had local hematoma that didn't need
Table1 1. Complications among 100 patients underwent percutaneous dilatational tracheostomies (PDT) in Tabriz city

\begin{tabular}{ll}
\hline Complications & n (\%) \\
\hline Serious complications & \\
Cardiopulmonary arrest & $0(0.0)$ \\
Trachoesophageal fistula & $0(0.0)$ \\
Intermediate complications & $0(0.0)$ \\
Posterior tracheal wall perforation & $0(0.0)$ \\
Minor complications & \\
Bleeding & $4(4.0)$ \\
Tube misplacement & $0(0.0)$ \\
Emphysema & $0(0.0)$ \\
\hline
\end{tabular}

any intervention and only conservative treatment had done. We didn't found any other complications such as emphysema, infection, posterior wall of trachea perforation (Table 1). The main underlying causes for the patients were: neurologic $58 \%$, pulmonary disease $22 \%$, metabolic and hypoxic encephalopathy $15 \%$, rheumatologic problems $3 \%$, other problems $2 \%$ (Table 2).

Table 2. Underlying cause for patient's referred to the center for percutaneous dilatational tracheostomies (PDT), Tabriz, $\operatorname{Iran}(\mathrm{n}=100)$

\begin{tabular}{lcc}
\hline & $\%$ & Totally (\%) \\
\hline $\begin{array}{l}\text { Neurologic } \\
\quad \text { CVA (Swallowing impairment, ineffective cough, and prolonged intubation } \\
\text { traumatic injury to cervical cord) }\end{array}$ & 41 & 58 \\
$\quad \begin{array}{l}\text { Multitrauma and head trauma with long time disturbance of consciousness } \\
\text { and need to prolonged intubation }\end{array}$ & 4 & \\
$\quad$ Neuromuscular & 5 & \\
Guillain-Barré syndrome & 4 & 8 \\
Myasthenia gravis & 3 & \\
Multiple sclerosis & 1 & 22 \\
Pulmonary & 15 & \\
$\quad$ Respiratory failure and long-term ventilation due to COPD & 1 & \\
$\quad$ Status asmaticus & 3 & \\
$\quad$ Lung parenchymal disease & 3 & \\
$\quad$ Sepsis or multiple organ failure & 15 \\
Hypoxic and metabolic encephalopathy & 10 & \\
$\quad$ MI with CPR & 1 & \\
$\quad$ Cardiopulmonary arrest & 1 & \\
$\quad$ Electric shock & 3 & \\
$\quad$ Severe drug poisoning & & \\
Rheumatologic & 1 & \\
$\quad$ Behcet disease & 2 \\
Pulmonary Renal Disorders & 2 & \\
$\quad$ Ketheacidosis & 1 & \\
$\quad$ Lymphoma & 1 &
\end{tabular}

$\mathrm{MI}=$ myocard infarct; $\mathrm{CPR}=$ cardiopulmonary resuscitation 


\section{DISCUSSION}

During the last decades, it has been proven that the tracheostomy has popularities like reducing pulmonary dead space, easier weaning of patients from the ventilator, make the opportunity to clearing the pulmonary secretions caused various (pathological or non-pathological) conditions, and also improves the patient's comfort. ${ }^{(4,12,14)}$ One patient had massive bleeding that need to surgical intervention to control the bleeding, and 3 cases had mild bleeding without any need for further intervention; meanwhile, of these 3 cases, two patients had hematoma (total complication rate: $4 \%$ ). These complications occurred at the beginning of this study and with progression didn't shown these complication, so this method needs to expert team, and we suggest after doing at least about 5 to 8 tracheostomy under observation of skilled person he or she can do this procedure alone without major complications. The related complication rates of this procedure in several studies were significantly larger than those observed in this study, ranging from 8.9 to $>30 \%,{ }^{(3,4,14)}$ while the total complication in this study was found in $0.06 \%$ cases (6 patients).

There was another important aspect in this study that skin incision before insertion of the needle help to reducing the time of dilatation and probability of infection. Performing tracheostomy under bronchoscopy was safer than other methods and without major complications. The results of this study suggest that the present method perform bedside tracheostomy in patients who haven't any contra indication for PDT.

As previously reported the complications of PDT, such as bleeding, infection and hypoxia, are less common, but in this study all complications of PDT were among the minor complications. Most are complications are minor. In a study by Klotz et al. ${ }^{(12)}$ the perioperative complication rate was $6 \%$, with premature extubation as the most common problem. Early postoperative complications were found in 5\% of patients, with bleeding as the most common complication.
Tracheal stenosis was seen in only $1.6 \%$ of patients who survived their ICU stay and were subsequently decannulated. However, the rate of these complications is low and death rarely occurs in this regard. It is reported three cases of extensive posterolateral longitudinal tracheal laceration that all were managed successfully with surgical repair. ${ }^{(14)}$

PDT is becoming the preferred method for tracheostomy in many centres. However, the currently available evidence suggests that apart from the risk related to intrahospital patient transport, PDT is comparable to surgical tracheostomy (ST). With respect to other complications, PDT is associated with lower infection and cost but higher chances of tube block and difficulty in decannulation compared to ST. ${ }^{(15,16)}$

Bleeding is the most frequent complication in this method. As with all patients undergoing a surgery, it is paramount that the patient did not have a coagulopathy. We recommend that the platelet count, prothromb in time and partial thromboplastic time be measured before PDT in all patients.

Our study has some limitations. We collected data retrospectively, and our findings should be confirmed in prospective trials. And complications related to anticoagulants suspension were not present in our study. Future investigations might also include the detailed analysis of the operating room utilization resources benefit from saving charges and using free operating room time space for additional elective procedures, examination of delayed complications, length of ventilation, and length of hospital stay.

\section{CONCLUSION}

PDT reduced the frequency of incidence of infection and indicated lesser complication rate which was found in 4 patients (4\%). Performing PDT in ICU should be considered as the main procedure to be performing in elective tracheotomy in critically adult patients. But further studies are recommended in larger population and 
with evaluation of more related parameters in this regard.

\section{CONFLICT OF INTEREST}

There is no conflict of interest

\section{CONTRIBUTORS}

MEH conducted the research.KBA and $\mathrm{YH}$ wrote the manuscript. LN, AS and MR supervised the research. VH and MR corrected the manuscript. All authors read and approved the manuscript.

\section{REFERENCES}

1. Frost EA. Tracing the tracheostomy. Ann Otol Rhinol Laryngol 1976;85(5 Pt.1):618-24.

2. Nowak A, Kern P, Koscielny S, et al. Feasibility and safety of dilatational tracheotomy using the rigid endoscope: amulticenter study. BMC Anesthesiol 2017;7:17. doi: 10.1186/s12871-0170301-y.

3. Putensen C, Theuerkauf N, Guenther U, et al. Percutaneous and surgical tracheostomy in critically ill adult patients: a meta-analysis. Critical Care 2014;18:544. doi:10.1186/s13054-014-0544-7.

4. Brass P1, Hellmich M, Ladra A, et al. Percutaneous techniques versus surgical techniques for tracheostomy. Cochrane Database Syst Rev 2016; 7:CD008045. doi: 10.1002/14651858. CD008045. pub2.

5. Kiran S1, Eapen S1, Chopra V1. A comparative study of complications and long term outcomes of surgical tracheostomy and two techniques of percutaneous tracheostomy. Indian J Crit Care Med 2015;19:82-6. doi: 10.4103/0972-5229.151015.

6. Pilarczyk K, Carstens H, Heckmann J, et al. Safety and efficiency of percutaneous dilatational tracheostomy with direct bronchoscopic guidance for thoracic transplant recipients. Respir Care 2016;61:235-42. doi: 10.4187/respcare.04128.

7. Acharya D, Nanjangud P. Percutaneous tracheostomy. Ind J Resp Care 2014; 3:488-96.

8. Ciaglia P, Firsching R, Syniec C. Elective percutaneous dilatational tracheostomy: a new simple bedside procedure; preliminary report. Chest 1985;87:715-9,

9. Ciaglia P. Technique, complications, and improvements in percutaneous dilatational tracheostomy. Chest 1999;115:1229-30.

10. Gromann TW, Birkelbach O, Hetzer R. Balloon dilatational tracheostomy: initial experience with the Ciaglia Blue Dolphin method. Anesth Analg 2009;108:1862-6.

11. Susarla SM, Peacock ZS, Alam HB. Percutaneous dilatational tracheostomy: review of technique and evidence for its use. J Oral Maxillofac Surg 2012;70:74-82.

12. Klotz R, Klaiber U, Grummich K, et al. Percutaneous versus surgical strategy for tracheostomy: protocol for a systematic review and meta-analysis of perioperative and postoperative complications. Syst Rev 2015;4:105. doi: 10.1186/s13643-015-0092-5.

13. Rajajee V, Fletcher JJ, Rochlen LR, et al. Real-time ultrasound-guided percutaneous dilatational tracheostomy: a feasibility study. Crit Care 2011; 15:R67. doi: 10.1186/cc10047.

14. Byhahn C. Percutaneous tracheostomy: the Ciaglia techniques. In: Servillo G, Pelosi P,editors. Percutaneous tracheostomy in critically illpatients. New York: Springer International Publishing Switzerland;2016.p.37-41.

15. Higgins KM, Punthakee X. Meta-analysis comparison of open versus percutaneous tracheostomy. Laryngoscope 2007;117:447-54.

16. Mirski MA, Pandian V, Bhatti N, et al. Safety, efficiency, and cost-effectiveness of a multidisciplinary percutaneous tracheostomy program. Crit Care Med 2012;40:1827-34. 